07

\title{
Нагрузочные характеристики спин-трансферного наноосциллятора
}

\author{
(C) А.Р. Сафрин ${ }^{1,2}$, Н.Н. Удалов ${ }^{1}$, М.И. Бичурин ${ }^{2}$, Р.В. Петров ${ }^{2}$, \\ A.C. Татаренко ${ }^{2}$ \\ ${ }^{1}$ Национальный исследовательский университет „МЭИ“, Москва \\ ${ }^{2}$ Новгородский государственный университет им. Ярослава Мудрого, \\ Великий Новгород \\ E-mail: arsafin@gma
}

\section{Поступило в Редакцию 2 сентября 2016 г.}

Построено семейство нагрузочных и регулировочных характеристик спинтрансферного наноосциллятора при разных значениях постоянного тока через образец путем интегрирования уравнения Ландау-Лифшица-ГильбертаСлончевского. Проведено сравнение полученных зависимостей с приближенными, полученными с помощью укороченных уравнений по первой гармонике. Вычислено приближенное выражение для сопротивления нагрузки, обеспечивающего максимум отдаваемой осциллятором мощности в нагрузку.

DOI: 10.21883/PJTF.2017.06.44404.16471

Одним из перспективных направлений развития спинтроники является спиновый токоперенос [1,2] в наноразмерных магнитных мультислойных структурах. Возможными приложениями данного направления являются: магнитная оперативная память, сверхчувствительные магнитные сенсоры, а также устройства формирования колебаний СВЧ- и THz-диапазонов частот $[3,4]$. Основными недостатками металлических спин-трансферных наноосцилляторов (СТНО) являются низкая выходная мощность (от десятков пиковатт до единиц нановатт) генерируемых колебаний и высокие значения ширины спектральной линии (сотни мегагерц на частотах в единицы гигагерц), существенно ограничивающие применение данных устройств в области телекоммуникаций. На данный момент применяются различные способы повышения мощности СТНО, например, используются магнитные туннельные переходы с промежуточными слоями из мультиферроиков [5,6], что позволяет существенно повысить сопротивление образцов во внешних магнитном и электриче- 

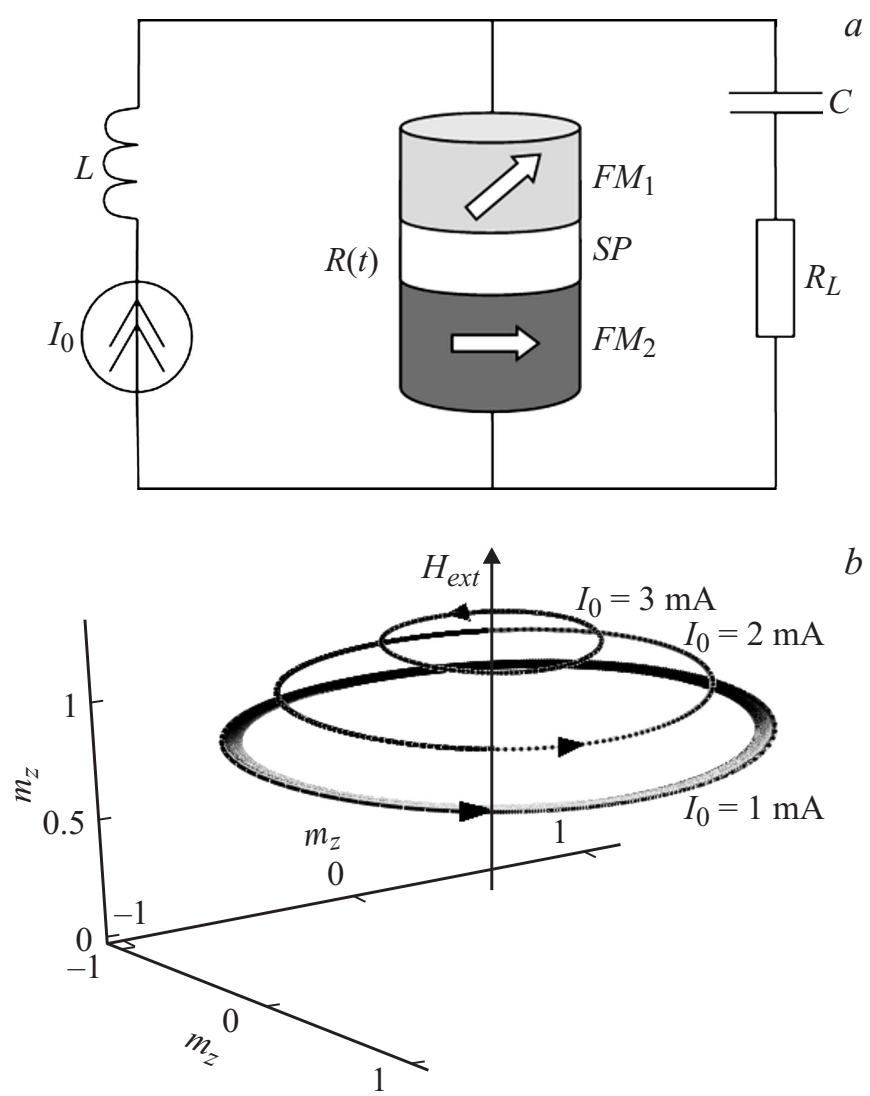

Рис. 1. $a-$ схема подключения СТНО с сопротивлением $R_{t}$ к нагрузке $R_{L}$ и идеальному источнику тока $I_{0} ; b-$ траектории системы (2) в фазовом пространстве $\left(m_{x}, m_{y}, m_{z}\right)$.

ском полях, а следовательно, и выходную мощность. Также интенсивно развивается направление взаимной и внешней синхронизации многих СТНО с целью сложения их мощностей в общей нагрузке [7-11]. В данной работе исследуются основные нагрузочные характеристики CTHО: мощность $P_{L}$ и частота $f_{\text {out }}$ выходных колебаний как функции сопротивления нагрузки $R_{L}$ и постоянного питающего тока $I_{0}$.

Письма в ЖТФ, 2017, том 43, вып. 6 
Схема подключения СТНО с переменным сопротивлением $R(t)$ к источнику тока $I_{0}$ и нагрузке $R_{L}$ через разделительную емкость $C$ и блокировочную индуктивность $L$ представлена на рис. $1, a$. СТНО представлен в виде туннельного перехода с двумя ферромагнитными слоями $F M_{1,2}$ и промежуточного слоя $S P$-спейсера. При пропускании тока через образец в слое с фиксированной намагниченностью $F M_{2}$ электроны поляризуются по спину и туннелируются в слой со свободной намагниченностью $F M_{1}$. За счет эффекта переноса спина $[1,2]$ при превышении тока некоторого критического значения намагниченность $\mathbf{m}=\mathbf{M} / M_{0}\left(M_{0}-\right.$ намагниченность насыщения $)$ слоя $F M_{1}$ начинает прецессировать в СВЧ-диапазоне. Сопротивление СТНО рассчитывается так [3]:

$$
R=\frac{R_{a p}+R_{p}}{2}-\frac{R_{a p}-R_{p}}{2}\left(\mathbf{m} \cdot \mathbf{e}_{p}\right)=R_{0}-\Delta R_{0}\left(\mathbf{m} \cdot \mathbf{e}_{p}\right),
$$

где $R_{a p}$ и $R_{p}-$ сопротивления СТНО при антипараллельной и параллельной конфигурациях намагниченностей слоев $F M_{1,2}, \mathbf{e}_{p}-$ единичный орт, задающий направление намагниченности слоя $F M_{2}$.

Математической моделью СТНО является [8] уравнение Ландау-Лифшица-Гильберта-Слончевского (ЛЛГС) для вектора $\mathbf{m}$ в следующем виде:

$$
\frac{d \mathbf{m}}{d t}=-\mu_{0}|\gamma|\left[\mathbf{m} \times \mathbf{H}_{e f f}\right]+\alpha\left[\mathbf{m} \times \frac{d \mathbf{m}}{d t}\right]+\sigma I\left[\mathbf{m} \times\left[\mathbf{m} \times \mathbf{e}_{p}\right]\right],
$$

где $\mathbf{H}_{e f f}=\mathbf{H}_{e x t}+\mathbf{H}_{m s}-$ эффективное магнитное поле, включающее внешнее магнитное поле $H_{e x t}$, направленное под углом $\theta_{e x t}$ к вертикали, поле размагничивания $\mathbf{H}_{m s}$ (с коэффициентами $N_{x, y, z}$ ); $\alpha$ - постоянная затухания Гильберта, константа $\sigma=\varepsilon g \mu_{\mathrm{B}} / 2 e M_{0} V, \varepsilon \approx 0.1-$ константа спиновой поляризации, $g-$ фактор Ланде, $\mu_{\mathrm{B}}-$ магнетон Бора, $e-$ заряд электрона и $V=S h$ - объем свободного слоя, $S$ - площадь поперечного сечения и $h$ толщина слоя $F M_{1}$. Ток $I$ через образец равен $I=\frac{R_{L}}{R_{L}+R} I_{0}$, а ток через нагрузку соответственно $I_{L}=\frac{R}{R_{L}+R} I_{0}$. Мгновенная мощность, выделяемая на нагрузке $p_{\text {out }}=I_{L}^{2} R_{L}$. На рис. $1, b$ представлено семейство фазовых траекторий системы (2) в фазовом пространстве $\left(m_{x}, m_{y}, m_{z}\right)$ в режиме генерации (величины токов $\left.I_{0}=1,2,3 \mathrm{~mA}\right)$ для следующих параметров: $\mu_{0} H_{e x t}=1.25 \mathrm{~T}$, $\theta_{\text {ext }}=0, \quad N_{x}=0.047, \quad N_{y}=0.072, \quad N_{z}=0.881, \quad V=3 \times 50 \times 75 \mathrm{~nm}^{3}$, 
$\alpha=0.02, \gamma_{p}=0, M_{0}=800 \mathrm{kA} / \mathrm{m}, R_{0}=450 \Omega, \Delta R_{0}=150 \Omega, R_{L}=\infty$. При превышении током $I_{0}$ некоторого критического значения в системе (2) наблюдается бифуркация Андронова-Хопфа рождения предельного цикла при потере устойчивости фокуса. Строгий анализ бифуркаций системы (2) представлен, например, в [4] и здесь не приводится. Далее сравним зависимости, получаемые численно интегрированием уравнения (2), с приближенными, полученными ниже.

Для приближенных количественных оценок от уравнения (2) перейдем к уравнению относительно комплексной амплитуды спиновой волны (укороченное уравнение по первой гармонике) свободного слоя $\dot{c}(t)=\left(m_{x}-i m_{y}\right) / \sqrt{2\left(1+m_{z}\right)}$ (см. подробнее [8]) в следующем виде:

$$
\frac{d \dot{c}}{d t}+i\left(\omega_{0}+N|\dot{c}|^{2}\right) \dot{c}+\Gamma_{G}\left(1+Q|\dot{c}|^{2}\right) \dot{c}-\sigma I\left(1-|\dot{c}|^{2}\right) \dot{c}=0,
$$

где $\omega_{0}$ - частота ферромагнитного резонанса СТНО; $N-$ коэффициент, характеризующий степень зависимости частоты колебаний от мощности спиновой волны (неизохронность); $\Gamma_{G}=\alpha \omega_{0}-$ коэффициент спин-волновых положительных потерь; $Q$ - коэффициент, характеризующий положительное нелинейное затухание. Поскольку $|\mathbf{m}|=1$, то $I \approx \frac{R_{L}}{R_{L}+R_{0}+\Delta R_{0}} I_{0}=\beta_{R} I_{0}$. Тогда выражение для стационарной мощности спиновой волны $p_{0}=\left|\dot{c}_{0}\right|^{2}=\lim _{t \rightarrow \infty}|\dot{c}|^{2}$ запишем так:

$$
p_{0}=\frac{I_{0} \beta_{R}-I_{t h}}{I_{0} \beta_{R}+Q I_{t h}} .
$$

Здесь $I_{t h}=\Gamma_{G} / \sigma-$ критический ток, при котором стартуют колебания в СТНО без влияния нагрузки.

Запишем выражение для частоты $\omega$ стационарных колебаний СТНО из (3)

$$
\omega_{\text {out }}=\omega_{0}+N \frac{I_{0} \beta_{R}-I_{t h}}{I_{0} \beta_{R}+Q I_{t h}} .
$$

Оценим величину мощности $P_{L}$ CТНО в нагрузке по первой гармонике. Первую гармонику тока $I_{L}^{(1)}$ приближенно оценим так (амплитуда первой гармоники разложения $\left(\mathbf{m} \times \mathbf{e}_{p}\right)$ равна $\left.\sqrt{p_{0}}\right)$ :

$$
I_{L}^{(1)} \approx \frac{\Delta R_{0} I_{0}}{R_{L}+R_{0}+\Delta R_{0}} \sqrt{p_{0}} .
$$

Письма в ЖТФ, 2017, том 43, вып. 6 
Тогда из (4) и (6) получаем следующее выражение для мощности $P_{L}$ :

$$
P_{\text {out }}=\frac{1}{2}\left(I_{L}^{(1)}\right)^{2} R_{L}=\frac{\Delta R_{0}^{2} \beta_{R} I_{0}^{2}}{2\left(R_{L}+R_{0}+\Delta R_{0}\right)^{2}} \frac{I_{0} \beta_{R}-I_{\text {th }}}{I_{0} \beta_{R}+Q I_{\text {th }}} .
$$

Минимальные значения тока $I_{0}$ и сопротивления нагрузки $R_{L}$, при которых стартуют колебания, определим из (7)

$$
I_{0}^{\min }=I_{t h} / \beta_{R}, \quad R_{L}^{\min }=\left(R_{0}+\Delta R_{0}\right) I_{t h} /\left(I_{0}-I_{t h}\right) .
$$

На рис. 2, $a$ представлены зависимости мощности по первой гармонике $P_{\text {out }}$ от сопротивления нагрузки $R_{L}$ для двух значений тока, построенные по (7). Точками обозначены результаты моделирования исходной системы (2). При увеличении значения сопротивления нагрузки от $R_{L}^{\min }$ зависимость $P_{\text {out }}\left(R_{L}\right)$ нарастает, достигая максимума при $R_{L}=R_{L}^{\text {out }}$ и далее убывает. Режим согласования, соответствующий максимуму отдаваемой в нагрузку мощности, соответствует $R_{L}=R_{L}^{\text {out }}$. Для его нахождения необходимо найти производную $\partial P_{\text {out }} / \partial R_{L}$ и приравнять получившееся выражение нулю. В общем случае эта задача сводится к кубическому уравнению и аналитическое выражение получается довольно громоздким. Однако при $Q \approx 0$ получаем довольно простое выражение

$$
R_{L}^{o p t}=\frac{I_{0}+I_{t h}}{I_{0}-I_{t h}}\left(R_{0}+\Delta R_{0}\right),
$$

которое для рис. $1, a$ при $I_{0}=6.5 \mathrm{~mA}$ и $I_{0}=8 \mathrm{~mA}$ дает $R_{L}^{o p t}=1.9 \mathrm{k} \Omega$ и $R_{L}^{o p t}=1.5 \mathrm{k} \Omega$ соответственно. На рис. $2, b$ при тех же значениях параметров представлены зависимости частоты выходных колебаний CTHO $f_{\text {out }}$ от сопротивления нагрузки $R_{L}$. Как видно, с увеличением сопротивления нагрузки частота выходных колебаний постепенно увеличивается.

На рис. 2, $c, d$ представлены регулировочные характеристики зависимости мощности $P_{\text {out }}$ и частоты $f_{\text {out }}$ выходных колебаний СТНО от величины постоянного тока $I_{0}$ для двух значений сопротивлений нагрузки: $R_{L}=0.5 \mathrm{k} \Omega$ и $R_{L}=1 \mathrm{k} \Omega$. Как следует из графиков, с ростом протекающего тока мощность и частота выходных колебаний увеличиваются, что согласовывается с численным интегрированием системы (2). Как известно из экспериментальных данных [8], увеличивать ток $I_{0}$ можно до значений примерно (4-5) $I_{t h}$. Дальнейшее увеличение тока может привести к выходу из строя СТНО.

Письма в ЖТФ, 2017, том 43, вып. 6 

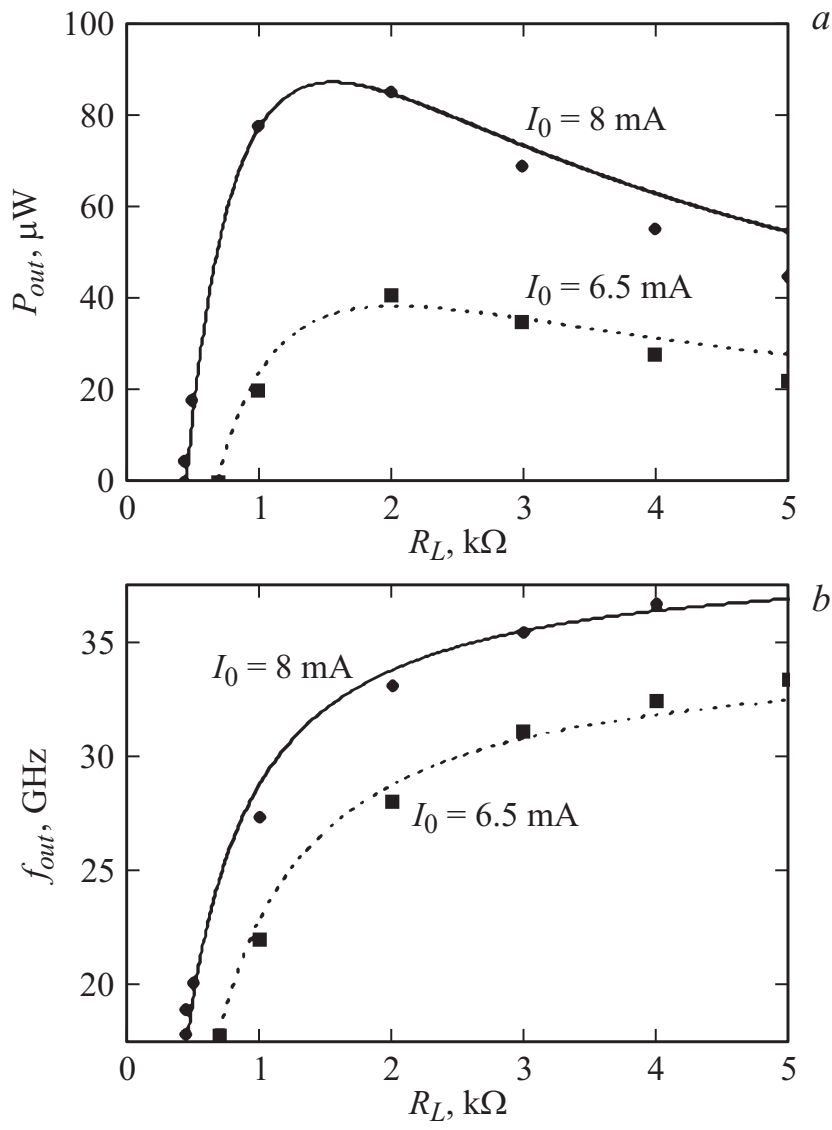

Рис. 2. Зависимости $P_{\text {out }}(a)$ и $f_{\text {out }}(b)$ от $R_{L}$ для $I_{0}=6.5 \mathrm{~mA}$ и $I_{0}=8 \mathrm{~mA}$; зависимости $P_{\text {out }}(c)$ и $f_{\text {out }}(d)$ от $I_{0}$ для $R_{L}=0.5 \mathrm{k} \Omega$ и $R_{L}=1 \mathrm{k} \Omega$.

Таким образом, построены и исследованы нагрузочные характеристики СТНО как с помощью интегрирования исходной системы уравнений ЛЛГС (2), так и с помощью приближенной автоколебательной модели Славина-Тиберкевича для комплексной амплитуды спиновой волны. Полученные приближенные зависимости (5) и (7) позволяют количественно оценить мощность и частоту выходных колебаний как

Письма в ЖТФ, 2017, том 43, вып. 6 

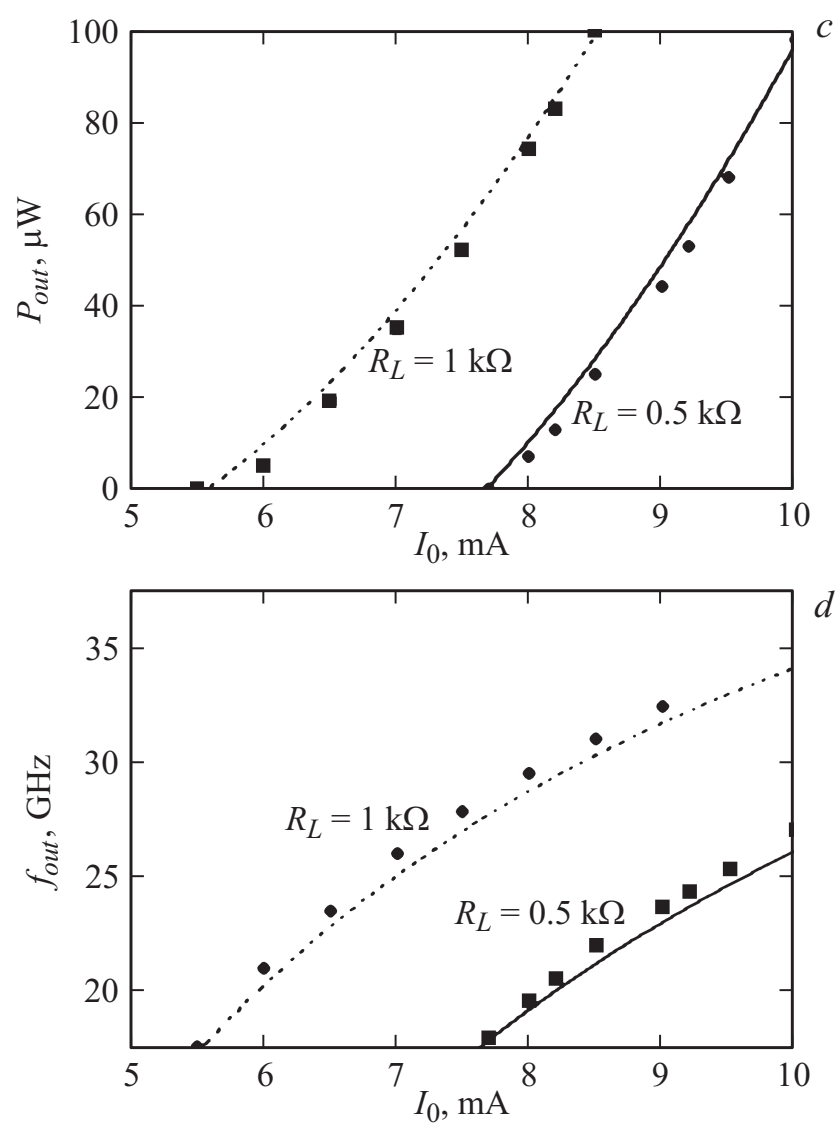

Pис. 2 (продолжение).

функции сопротивления нагрузки и тока. Выражение (9) позволяет при конструировании усилителя мощности для СТНО количественно найти сопротивление нагрузки в режиме согласования, т.е. при максимуме отдаваемой в нагрузку мощности. Развитый в данной работе подход может также применяться для определения режима согласования в спин-трансферных осцилляторах вихревого типа, не использующих внешнее магнитное поле $[12,13]$.

Письма в ЖТФ, 2017, том 43, вып. 6 
Работа выполнена при финансовой поддержке гранта РНФ № 15-19-10036.

\section{Список литературы}

[1] Slonczewski J. // J. Magn. Magn. Mater. 1996. V. 159. P. L1.

[2] Berger L. // Phys. Rev. B. 1996. V. 54. P. 9353-9358.

[3] Villard P. et al. // IEEE J. Solid-State Circ. 2010. V. 45. N 1. P. 214-223.

[4] Корнеев В.И. и др. //ФТТ. 2009. Т. 51. В. 1. С. 118-128.

[5] Velev J. et al. // Phil. Trans. R. Soc. A. 2011. V. 369. P. 3069-3097.

[6] Gajek M. et al. // Nat. Mat. 2007. V. 6. P. 296-302.

[7] Grollier J. et al. // Phys. Rev. B. 2006. V. 73. P. 060409.

[8] Slavin A., Tiberkevich V. // IEEE Trans. Magn. 2009. V. 45. N 4. P. 1875-1918.

[9] Safin A. et al. // Eur. Phys. J. Appl. Phys. 2014. V. 67. P. 20601.

[10] Митрофанов А. и др. // Письма в ЖТФ. 2014. Т. 40. В. 13. С. 66-72.

[11] Митрофанов А. и др. // Письма в ЖТФ. 2015. Т. 41. В. 16. С. 29-35.

[12] Tsunegi S. et al. // Appl. Phys. Exp. 2014. V. 7. P. 063009.

[13] Dussaux A. et al. // Appl. Phys. Lett. 2014. V. 105. P. 022404.

Письма в ЖТФ, 2017, том 43, вып. 6 